\title{
Numerical Studies of Abrasion Wear on the Guide Vanes in a Submersible Axial Flow Pump
}

\author{
H.M. Zhang \\ Department of Engineering Mechanics \\ Kunming University of Science and Technology \\ Kunming , P. R. China
}

\author{
L.X. Zhang \\ Department of Engineering Mechanics \\ Kunming University of Science and Technology \\ Kunming , P. R. China
}

\begin{abstract}
The paper presents the numerical studies of abrasion wear on the guide vanes in a submersible axial flow using OpenFOAM code, which is an Open Source CFD Package. Hashish erosion model was implemented in this code. The 3-D turbulent particulate-liquid two-phase flow equations are employed in this study. The computing domain is discretized with a full three-dimensional mesh system of unstructured tetrahedral shapes. The finite volume method is used to solve the governing equations and the pressure-velocity coupling is handled via a Pressure Implicit with Splitting of Operators (PISO) procedure. Simulation results have shown that the sand erosion rate on pressure side is more than on the suction side of the guide vanes. The abrasion wear occurs mainly at the lower part of the guide vanes.
\end{abstract}

Keywords- CFD; Abrasion wear; Submersible axial flow pump; open FOAM

\section{INTRODUCTION}

A submersible axial flow pump often used in sewage pumping station. Due to sewage contains large amounts of sediment, solids and other impurities, the components of axial flow bump will be worn and results in reduced efficiency. The abrasion wear is due to the dynamic action of sediment or solid particles flowing along with water impacting against a solid surface of hydraulic components. In general, a number of factors influence the development of abrasion wear process of hydraulic machinery. These factors include mean velocity of particles, mass of the particle, concentration of the abrasive particles in a liquid flow, grain size and shape of the particles and angle of attack at which the particles collide with the surface etc. With the aid of CFD, numerical solutions for the equations governing fluid flows can be successfully obtained in order to accurately predict the different outcomes of fluid-surface interactions. Meng and Ludema[1] separated 28 erosion models out of the almost 2000 empirical models they encountered and concluding that each equation is the result of a very specific and individual approach. López at al. [2] compared the different erosion models in an Eulerian-Lagrangian frame using OpenFoam. They believe that Hashish's model for erosion would be a reasonable approach when experimentation is not feasible. They analyzed the erosion rates of a single channel of a centrifugal pump subjected to solid particle impingement using four different methods with OpenFoam [3]. Results yielded some disparities between models due to the different factors taken into consideration.
This paper presents using OpenFOAM code only to make a qualitative analysis of the abrasion wear on the guide vanes in a submersible axial flow pump. OpenFOAM is an object-oriented $\mathrm{C}++$ library of classes and routines of use for writing CFD codes. It has a set of basic features similar to any commercial CFD solver, such as turbulence models and discretization schemes. With OpenFOAM it is easy to add any modification to any part of the implementation. Hashish erosion model was implemented here.

\section{GOVERNING EQUATIONS}

The flow model used for the numerical simulations is based on the generalized homogeneous multiphase flow model with the additional sources of momentum for the effects of the Coriolis and centrifugal accelerations in a steady rotating frame of reference. The governing equations are described below[4]

Continuity equation of liquid:

$$
\frac{\partial}{\partial t}(\rho)+\frac{\partial}{\partial x_{j}}\left(\rho \boldsymbol{u}_{j}\right)=S=-\sum n_{p} \dot{m}_{p}
$$

Momentum equations of liquid:

$$
\begin{aligned}
& \frac{\partial\left(\rho \boldsymbol{u}_{i}\right)}{\partial t}+\frac{\partial}{\partial x_{j}}\left(\rho \boldsymbol{u}_{j} \boldsymbol{u}_{i}\right)=-\frac{\partial p}{\partial x_{i}}+\frac{\partial}{\partial x_{j}}\left[\mu_{e}\left(\frac{\partial u_{i}}{\partial x_{j}}+\frac{\partial u_{j}}{\partial x_{i}}\right)\right] \\
& +\sum \rho_{p} C_{D} \frac{3}{4} \frac{R_{e p} \mu}{\bar{\rho}_{p} d_{p}^{2}}\left(\boldsymbol{u}_{p i}-\boldsymbol{u}_{i}\right) \\
& +\sum C_{D} \frac{3}{4} \frac{R_{e p} \mu}{\bar{\rho}_{p} d_{p}^{2}}\left(\frac{v_{p}}{\sigma_{p}} \frac{\partial \rho_{p}}{\partial x_{j}}\right)+F_{c j}
\end{aligned}
$$

$$
\begin{gathered}
\text { Where } F_{c j} \quad \text { represents the } \\
\text { force. } P=P^{*}+0.5 \rho(\omega r)^{2} \text { is total pressure, } P^{*} \text { is static }
\end{gathered}
$$
pressure, $\rho_{p}$ is the volume density of the particle phase, $n_{p}$ is the number of particles per unit volume, $m_{p}$ is the particle mass, $C_{D}$ is the drag coefficient of unsteady flow.

Continuity equation of particle: 


$$
\frac{\partial}{\partial t}(\rho)+\frac{\partial}{\partial x_{j}}\left(\rho \boldsymbol{u}_{j}\right)=\frac{\partial}{\partial x_{j}}\left(\frac{v_{p}}{\sigma_{p}} \cdot \frac{\partial \rho_{p}}{\partial x_{j}}\right)
$$

Momentum equations of particle:

$$
\begin{aligned}
& \frac{\partial\left(\rho_{p} \boldsymbol{u}_{p i}\right)}{\partial t}+\frac{\partial}{\partial x_{j}}\left(\rho_{p} \boldsymbol{u}_{p j} \boldsymbol{u}_{p i}\right)=\frac{\partial}{\partial x_{j}}\left[\rho_{p} \boldsymbol{u}_{p}\left(\frac{\partial u_{p i}}{\partial x_{j}}+\frac{\partial u_{p j}}{\partial x_{i}}\right)\right] \\
& +\sum \frac{m_{p} C_{D} \frac{3}{4} \frac{R_{e p} \mu}{\bar{\rho}_{p} d_{p}^{2}}+m_{p}}{m_{p}} \frac{v_{p}}{\sigma_{p}} \frac{\partial \rho_{p}}{\partial x_{i}} \\
& +\frac{\partial}{\partial t}\left(\frac{v_{p}}{\sigma_{p}} \frac{\partial \rho_{p}}{\partial x_{i}}\right)+F_{p c i}+F_{p l i}+F_{p p i}
\end{aligned}
$$

Where $F_{p c i}$ is the Coriolis force, $F_{p l i}$ is the particles centrifugal force, $F_{p p i}$ is the particle phase pressure.

\section{Erosion model:}

Hashish erosion model is used here [5]. It modified Finnie's model for erosion by solid particle impingement in ductile materials taking into account particle shape and including no empirical constants. The equation, taken from [2], and used for the simulation is equation (5).

$$
W=\frac{7}{\pi} \frac{M}{\rho_{p}}\left(\frac{V}{C_{k}}\right)^{2.5} \sin (2 \alpha) \sqrt{\sin \alpha}
$$

Where $M$ is the mass of the abrasive particles, $\rho_{p}$ is the density of the particles, $V$ is the velocity magnitude of the particles, $\alpha$ is the angle of impingement, $C_{k}$ is a coefficient which can be obtained from equation (6).

$$
C_{K}=\sqrt{\frac{3 \sigma_{f} R_{f}^{\frac{2}{5}}}{\rho_{p}}}
$$

Where $R_{f}$ is the roundness factor of the particulate phase.

\section{Numerical Algorithm}

In the OpenFOAM , the spatial discretization is performed using a cell centered co-located finite volume method for unstructured meshes with arbitrary cell-shapes, and a multi-step scheme are used for the time derivatives. For the simulations presented in this paper, a second order implicit time scheme is used combined with second order linear interpolation in space, except for the convective terms. The time step is equal to 1 degrees of runner revolution. The iterative solvers are considered converged when the residuals have been reduced by a factor of 10-5. The pressure-velocity coupling is handled via a Pressure Implicit with Splitting of Operators (PISO) procedure. The $k-\omega$ SST turbulence model is adopted for simulation of the unsteady flow through a submersible axial pump. It has been implemented in the OpenFOAM libraries.

\section{Grid, Boundary and Initial Conditions}

Figure 1 shows the calculation domain of the submersible axial flow pump. The parameters are listed in Table 1.

The computational grid of the complete axial flow pump is generated using ICEM CFD, including inlet, guide vane, impeller and outlet with 23,600,000 elements was used. After importing the meshed geometry in OpenFOAM, it is divided into four domains namely inlet, vanes, impeller and outlet, in which impeller is rotating domain and others are stationary domains. The element number of impeller domain is 13,600,000.

In the algorithm, the length of the time step was equal to one of the impeller revolution. Rotation speed of the runner was 580 $\mathrm{r} / \mathrm{min}$. Thus, the time step was $0.0036236 \mathrm{~s}$. Inlet bulk mass flow rate and fixed outlet pressure boundary conditions are applied. The calculation is done on a SUGON high-performance computers using $160 \mathrm{CPU}$ core.

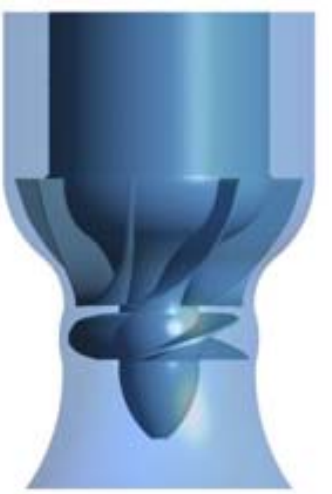

FIGURE I. SUBMERSIBLE AXIAL FLOW PUMP

TABLE I. PARAMETER OF SUBMERSIBLE AXIAL PUMP

\begin{tabular}{|l|l|}
\hline Diameter of impeller $(\mathrm{m})$ & 0.165 \\
\hline Number of impeller blade & 3 \\
\hline Number of stay vane & 8 \\
\hline head $(\mathrm{m})$ & 5.85 \\
\hline Rotational speed $(\mathrm{rpm})$ & 580 \\
\hline Rated flow rate $\left(\mathrm{m}^{3} / \mathrm{s}\right)$ & 0.981 \\
\hline
\end{tabular}

\section{ANALYSIS OF NUMERICAL CALCULATION RESUlTS}

The sand volume fractions and the sand erosion rate are the important feature for abrasion wear in hydraulic turbine. The region of higher sand volume fraction and sand erosion rate with the region of abrasion wear is consistent qualitatively. Figures 2 show the pressure distribution for the guide vanes on the pressure side and section side. The pressure is relatively high at the lower part of the guide vanes. Figures 3 show the volume fraction of sand distribution for the guide vanes on the pressure side and the section side. It is clearly seen that the sand volume 
fraction at the lower part of the pressure side is higher than others. Compared with the sand erosion rate distribution of Figure 4, the maximum of sand volume fraction is at same location with the maximum of sand erosion rate. Figures 5 show the wall stress distribution for the guide vanes on the pressure side and section side. Its maximum position is also consistent with the maximum position of the sand erosion rate and sand volume fraction. Figures 6(a) show the sand erosion rate distribution on guide vanes and Figures 6(b) show the sand volume fraction distribution on guide vanes. The sand erosion rate and sand volume fraction in the lower part of the guide vane is relatively high.
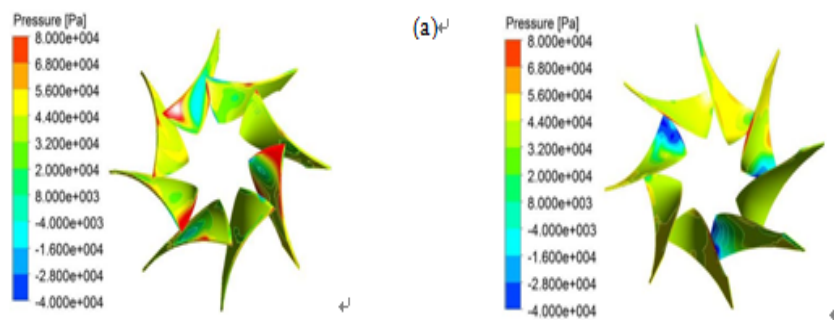

FIGURE II. THE PRESSURE DISTRIBUTION FOR THE GUIDE VANES ON THE PRESSURE SIDE AND SECTION SIDE
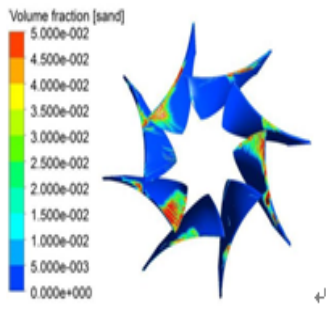

(a)

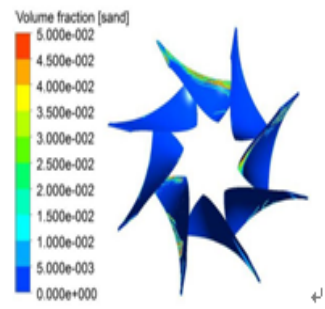

FIGURE III. THE SAND VOLUME FRACTION DISTRIBUTION FOR THE GUIDE VANES ON THE PRESSURE SIDE AND SECTION SIDE

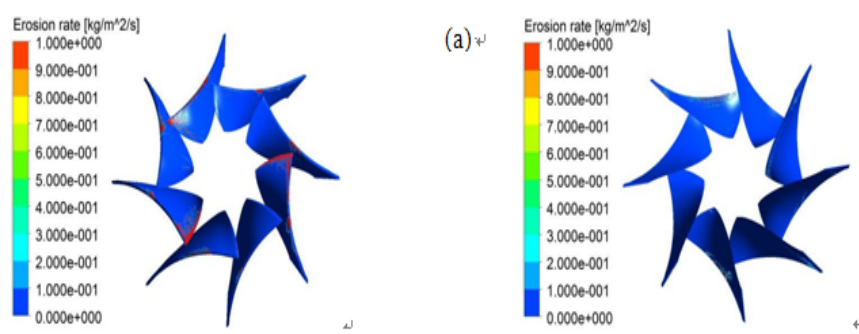

FIGURE IV. THE SAND EROSION RATE DISTRIBUTION FOR THE GUIDE VANES ON THE PRESSURE SIDE AND SECTION SIDE
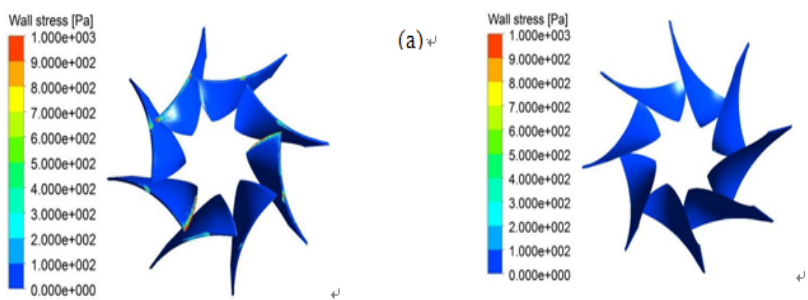

(b)

FIGURE V. THE WALL STRESS DISTRIBUTION FOR THE GUIDE VANES ON THE PRESSURE SIDE AND SECTION SIDE

(b) 4

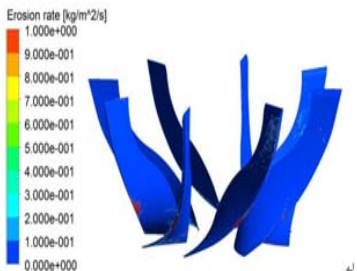

(a)

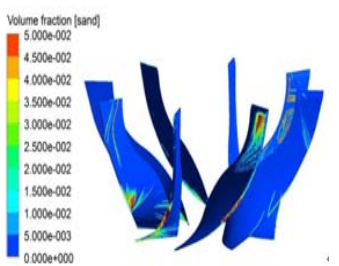

(b)
FIGURE VI. (a) THE SAND EROSION RATE DISTRIBUTION ON GUIDE VANES (b) THE SAND VOLUME FRACTION DISTRIBUTION ON GUIDE VANES

\section{CONCLUSION}

In this paper, the numerical studies of abrasion wear on the guide vanes in a submersible axial flow pump has been carried. Simulation results have shown that the sand erosion rate on pressure side is more than on the suction side of the guide vanes. The abrasion wear occurs mainly at the lower part of the guide vanes. Hashish erosion model implemented in OpenFoam is able to simulate the wear problem well.

\section{ACKNOWLEDGEMENTS}

This work was financially supported by the National Natural (b) Science Foundation of china (No. 51279071) and the Natural Science Foundation of Kunming University of Science and Technology (No.20149x16).

\section{REFERENCES}

[1]. Meng H C, Ludema K C. Wear models and predictive equations: their form and content[J]. Wear, 1995, 181: 443-457.

[2]. López A, Stickland M T, Dempster W M. Comparative study of different erosion models in an Eulerian-Lagrangian frame using Open Source software[J].

[3]. Lopez A, Stickland M, Dempster W. Modeling erosion in a centrifugal pump in an Eulerian-Lagrangian frame using OpenFOAM ${ }^{\circledR}[\mathrm{J}]$.

[4]. Tang Xue-Lin, TANG Hong-Fen,WU Yu-Lin. Simulating and erosion prediction of 3D two phase flow through a turbine runner,JOURNAL OF ENGINEERING THERMOPHYSICS, Vol.22, N0.1, jan.,2001.

[5]. Hashish M. Modified model for erosion. Proceedings of the 7th International Conference on Erosion by liquid and solid impact, 1987 Cambridge, UK, pp. 461-480 